Introduction Low HIV serostatus awareness and delayed treatment initiation among people living with HIV (PLHIV) contribute to HIV sexual transmission. An unacceptably low proportion of PLHIV are aware of their HIV status in Latin America. Improved understanding of sexual risk among PLHIV could help guide HIV prevention strategies.

Methods A cohort of 401 men who have sex with men (MSM) and transgender women at high risk were enrolled and assessed every three months for condomless sex and sexually transmitted infections (i.e. syphilis, HIV and anal chlamydia, and anal gonorrhoea). Among those who were positive at entry, we compared condomless sex and anal chlamydia/gonorrhoea according to prior knowledge of HIV serostatus; among those who seroconverted during follow-up, we compared condomless sex and anal chlamydia/gonorrhoea before vs. after HIV diagnosis, using McNemar's Chi-square test.

Results At baseline, 82 (20.5\%) participants self-identified as HIV positive and an additional $42(10.5 \%)$ were diagnosed with HIV. Among the 42 unknown HIV positives, 71\% reported recent condomless receptive anal sex compared to 55\% of known HIV positives ( $\mathrm{p}$-value $=0.078$ ). No difference was observed in condomless insertive anal sex; $48 \%$ in each group. Among the 24 sero-converters during follow-up, 79\% reported condomless receptive anal sex prior to their diagnosis and $32 \%$ after their diagnosis $(59 \%$ decline, p-value $=0.001) ; 46 \%$ reported condomless insertive anal sex prior to their diagnosis and $14 \%$ after diagnosis $(70 \%$ decline, $\mathrm{p}$-value $=0.011)$. Anal gonorrhoea and/or chlamydia were diagnosed among $46 \%$ prior and $27 \%$ after diagnosis among the observed sero-converters (41\% decline, $\mathrm{p}$-value $=0.096$ )

Conclusions Risk behaviours and concurrent STIs diminish after a new diagnosis, following the patterns previously reported elsewhere. Current prevention efforts for PLHIV are insufficient and must take into account motivations for sexual risk taking, encourage people to reach viral suppression, and improve available prevention strategies to prevent onward transmission of HIV.

Disclosure of interest statement None.

\section{P16.04 CLINICO-EPIDEMIOLOGICAL PROFILE OF ELDERLY HIV PATIENTS ATTENDING ANTI RETRO-VIRAL THERAPY (ART) CENTRE OF A TEACHING HOSPITAL IN SOUTH INDIA}

D Madi*, N Ramakrishnan, J Ramapuram. Department of Medicine, KMC Mangalore (Manipal University)

\subsection{6/sextrans-2015-052270.551}

Introduction Human Immunodeficiency Virus (HIV) infection was previously thought as a disease of the young. Clinicians are now encountering increasing numbers of older HIV patients in routine practice. Management elderly HIV can be challenging as they may present with advanced disease and they may also have multiple co-morbidities. There is a dearth of research in this field from India. The aim of our study was to determine the clinico-epidemiological profile of elderly ART naïve HIV patients attending a tertiary care institution.

Methodology This cross-sectional study was done in the ART centre attached to a tertiary care hospital of Southern India. Data of 120 patients who were diagnosed to be HIV-positive at or after age of 50 years (elderly HIV) from 2009 to 2014 was analysed after approval from the ethics committee.
Sociodemographic and clinical characteristics were described using descriptive statistics.

Results Among 786 HIV patients detected from 2009 to 2014, $120(15.27 \%)$ were elderly HIV. Majority of them 82 (68.33\%) were males. The mean age of males and females was $56.12 \pm$ 6.88 and $55.34 \pm 4.23$ years. Heterosexual mode was the commonest mode of acquiring HIV 74 (61.67\%). Majority of them $77(64.17 \%)$ were in WHO stage 1. Tuberculosis was seen in 32 (26.6\%) The common co-morbidities seen were hypertension 93 (77.5\%) and diabetes 8 (6.6\%). The median CD4 count $(\mathrm{n}=$ 112) at presentation was 245 cells $/ \mathrm{mm}^{3}$ (IQR 145-426.2). Late presenters (CD4 less than 200 cells $/ \mathrm{mm}^{3}$ ) were 47 (41.96\%).

Conclusion In our study $15 \%$ of patients attending ART centre were elderly. Majority of them had co morbidities. A significant proportion of them were late presenters. Physicians should have a high index of suspicion in diagnosing HIV in this age group as most of the symptoms of this disease may simulate the process of normal ageing. National programmes and policy makers must focus their attention on geriatric HIV.

\section{P16.05 REGIONAL EPIDEMIOLOGICAL PATTERN OF AIDS- RELATED CANCERS IN CHILDREN}

${ }^{1,2} \mathrm{O}$ Adetokunboh*, ${ }^{1} \mathrm{~T}$ Balogun. 'Stellenbosch University, South Africa; ${ }^{2}$ Centre for Healthcare Research and Training, Nigeria

\subsection{6/sextrans-2015-052270.552}

Introduction Individuals living with human immunodeficiency virus (HIV)/acquired immunodeficiency syndrome (AIDS) are vulnerable to develop certain malignancies such as Non-Hodgkin lymphoma, Kaposi's sarcoma and cervical cancer. Cancers may not be very common among the paediatric age group, however it is of significant importance among HIV-infected children. This study evaluates incidence and mortality patterns of the three AIDS-related cancers in the six World Health Organization (WHO) regions.

Methods The study data was accessed from the International Agency for Cancer Research GLOBOCAN 2012 database. Incidence and mortality rates for children aged $0-14$ years old using age-specific rates and numbers.

Results African region had the highest number of NHL and KS new cases [38\% (6296/16509)] and KS [96\% (2081/2162)] respectively while Western Pacific region had 41\% (68/165). The regions recorded 18,836 new cases (NHL - 88\%, KS -11\% and cervical cancer - 0.9\%) while the mortality cases followed almost the same pattern. The total number of new cases of NHL for female was 5885, 10624 for the male $(\mathrm{P}=0.1668)$, and that of KS was 963 for female and 1199 for male $(\mathrm{P}=0.8757)$. Africa recorded the highest incidence rates for NHL $(1.3 / 100$ 000 for female and 2.1/100 000 for male). The region also recorded the highest mortality rates for NHL $(0.7 / 100,000$ for female) and 1.0/100,000 for male). The situation was the same with KS with the African region having the highest incidence and mortality rates for both gender.

Conclusions The distribution of Non-Hodgkin lymphoma, Kaposi's sarcoma and cervical cancer followed the pattern of HIV prevalence in the WHO regions. Africa being the most affected region recorded the highest incidence and mortality in children (both HIV-infected and non - infected). There is no doubt that KS is predominantly an African problem while cervical cancer is rare among children despite the HIV epidemic. 
Disclosure of interest statement No grants were received in the development of this study.

\section{P16.06 KNOWLEDGE OF KAPOSI SARCOMA (KS) AMONG HIV + COMMERCIAL SEX WORKERS ON HIV THERAPY IN KISUMU TOWNSHIP, KENYA}

C Kiplagat*, M Mutai, E Sumukwa. Jaramogi Oginga Odinga Teaching Hospital in Kisumu, Kenya

\subsection{6/sextrans-2015-052270.553}

Introduction The most common type of KS in the United States is epidemic or AIDS-related KS. Kaposi Sarcoma is a type of malignancy which develops in people who are infected with HIV, the virus that causes AIDS. Studies have suggested that the prevalence rate of KS is low on HIV Positive Persons due to an excellent ART therapy and remains higher on HIV Negative Persons.

Methods We performed a retrospective analysis in all HIV infected patients having KS using Demographic and Clinical parameters. We used outpatient Clinic at Jaramogi Oginga Odinga Teaching and referral Hospital in Kisumu Township as our main hospital for this research between the years of 2009-2014 respectively.

Results Among 2280 patients, 30 were diagnosed with KS in the period of 2009-2014. All Patients were commercial sex workers with a mean age of 35 (30-38). Kaposi Sarcoma was Diagnosed in different stages of HIV infection by using Cohorts.

Cohort 1: KS concomitant Diagnosis of HIV infection were 6 cases

Cohort II: Diagnosis of KS in cART naïve persons were 7 cases

Cohort III: Diagnosis of KS in Patients for the first 6 months of start of cART as IRIS were 4 cases

Conclusion All patients who were treated with 2NRTI+PI were permanently cured of KS. Also there was no KS cases observed on the stable and virological effective cART.

\section{P16.07 PRIOR CONDYLOMA AND SYPHILIS DIAGNOSIS AMONG HIV-INFECTED PERSONS: A NATIONWIDE POPULATION- BASED STUDY IN TAIWAN, 2000-2010}

${ }^{1} \mathrm{C}$ Strong ${ }^{*},{ }^{2} \mathrm{H}$ Zou, ${ }^{3} \mathrm{Y}$ Chen, ${ }^{1} \mathrm{C} \mathrm{Li}, 4,5 \mathrm{~W}$ Ko, ${ }^{4,6} \mathrm{~N} \mathrm{Ko}$. 'Department of Public Health, National Cheng Kung University, Tainan, 701, Taiwan; ${ }^{2}$ Kirby Institute, University of New South Wales, Sydney, 2052, Australia; ${ }^{3}$ Institute of Allied Health Sciences, College of Medicine, National Cheng Kung University, Tainan, 701, Taiwan; ${ }^{4}$ Department of Internal Medicine, National Cheng Kung University Hospital, Tainan, 701, Taiwan; ${ }^{5}$ College of Medicine, National Cheng Kung University, Tainan, 701, Taiwan; ${ }^{6}$ Department of Nursing, National Cheng Kung University, Tainan, 701, Taiwan

\subsection{6/sextrans-2015-052270.554}

Introduction Previously acquired sexually transmitted infections (STIs) increase the risk of HIV infection, yet few studies have examined the time interval between prior condyloma/syphilis diagnosis and HIV infection. We aimed to examine numbers of condyloma/syphilis diagnosis, and HIV screening prior to HIV infection in relation to HIV risk among HIV-infected persons. Methods This population-based study retrieved medical claims data from Taiwan National Health Insurance Research Database (NHIRD) from 2000 to 2010 and included 16119 HIV-infected persons more than 15 years of age. We included cases with prior condyloma/syphilis diagnosis before HIV infection and described the elapsed time between first condyloma/syphilis in the database and HIV infection. A subgroup analysis was conducted for those who have prior condyloma/syphilis diagnosis, using multivariable Cox proportional hazard regression to examine factors associated with HIV infection within 1 year.

Results One hundred and fifty-two HIV-infected persons received condyloma diagnosis prior to HIV infection with an average elapsed time of 644 days. Among them, 31\% became HIV-infected within one year after first diagnosis of condyloma in the database. For syphilis, 833 persons received syphilis diagnosis and the average elapsed time was 1025 days. Among them, $24 \%$ became HIV-infected within one year. For those who have been diagnosed with condyloma, each increment of condyloma diagnosis before HIV infection was associated with an 2.8 times of risk for HIV infection within 1 year $(95 \%$ confidence interval $(\mathrm{CI})=1.26-6.19)$. For those who were diagnosed with syphilis, numbers of condyloma and syphilis diagnosis and HIV screening were significantly associated with increased risk of HIV infection within 1 year, with adjusted hazard ratios (aHRs) of 3.97 (1.699.32), 1.95 (1.31-2.89), 1.72 (1.53-1.93), respectively.

Conclusion History of condyloma/syphilis before HIV infection was associated with risk of HIV infection among HIV-infected persons. More regular HIV screening among persons with STIs are warranted.

Disclosure of interest statement The authors declare that there are no conflicts of interest.

\section{P16.08 PREVALENCE OF SEXUALLY TRANSMITTED INFECTIONS AMONG PREGNANT HIV-POSITIVE IN CENTRAL-WEST BRAZIL}

SM Brunini ${ }^{1 *}$, J Valadares', LCC Guimarães ${ }^{2}$, BL Almeida2, CM Souza $2 .{ }^{1}$ Federal University of Goias, Brazil; ${ }^{2}$ Postgraduate Nursing Program of Federal University of Goias, Brazil

\subsection{6/sextrans-2015-052270.555}

Introduction In Brazil, the feminization of Human Immunodeficiency Virus epidemic (HIV) is a reality. This situation illustrates the occurrence of a period in a woman's life, pregnancy. In the HIV-positive co-infections are frequent, given that the virus shares the same transmission routes with other pathogens. In pregnant women, this condition is extremely worrying to consider the possibility of vertical transmission of HIV and other Sexually Trasmitted Infections (STIs) during pregnancy, childbirth or breastfeeding.

Methods A cross-sectional study, retrospective. Data collection was obtained from medical records. We reported the prevalence of other STIs in pregnant women living with HIV, met in the reference maternity of State Goias, Brazil, from January 2007 to December 2013.

Results Were evaluated 323 pregnant HIV-positive. Most women $(47,2 \%)$ were between 25 and 34 years of age, poorly educated $(74.2 \%)$ and reported being a housewife (75,3\%). 281 women $(79,6 \%)$ reported inconsistent condom use.

The overall prevalence of STDs was 14.5\% (95\% CI 11.02 18.72). Syphilis was detected in 5.0\% (95\% CI 2.96-7.75); Condylomatosis in $3.71 \%$ (95\% CI 2.13-6.38); Trichomonas vaginalis in $0.61 \%$ (95\% CI: $0.17-2.23)$. Serological markers of hepatitis B virus infection was present in 1.24\% (95\% CI: $0.48-$ 3.14). Vaginosis was also identified in $2.17 \%$ (95\% CI: $2.17-$ 4.40).

Conclusion The substancial prevalence of STIs including syphilis was found among pregnant women HIV-positive in the study. 